\title{
Chiral susceptibility in a dense thermomagnetic QCD medium within the hard thermal loop approximation
}

\author{
Ritesh Ghosh, ${ }^{*}$ Bithika Karmakar®, ${ }^{\dagger}$ and Munshi Golam Mustafa $\odot^{\ddagger}$ \\ Theory Division, Saha Institute of Nuclear Physics, 1/AF, Bidhannagar, Kolkata 700064, India \\ and Homi Bhabha National Institute, Anushaktinagar, Mumbai, Maharashtra 400094, India
}

(Received 10 December 2020; accepted 18 March 2021; published 23 April 2021)

\begin{abstract}
We compute the chiral susceptibility in quark-gluon plasma in the presence of a finite chemical potential and a weak magnetic field within hard thermal loop approximation. First we construct the massive effective quark propagator in a thermomagnetic medium. Then we obtain a completely analytic expression for the chiral susceptibility in the weak magnetic field approximation. In the absence of a magnetic field, the thermal chiral susceptibility increases in the presence of a finite chemical potential. The effect of the thermomagnetic correction is found to be very marginal as temperature is the dominant scale in a weak field approximation.
\end{abstract}

DOI: 10.1103/PhysRevD.103.074019

\section{INTRODUCTION}

It has been a long-standing quest of the heavy ion collision community to explore the phase diagram of QCD. Several large scale experiments such as the Large Hadron Collider (LHC) at CERN and the Relativistic Heavy Ion Collider (RHIC) at Brookhaven National Laboratory (BNL) have been designed and performed for this purpose. Upcoming experiments at Facility for Antiproton and Ion Research (FAIR), Nuclotron-based Ion Collider fAcility (NICA), Japan Proton Accelerator Research Complex (JPARC) are expected to examine the phase diagram of QCD at high baryon density. Two nonperturbative features of QCD vacuum are confinement and chiral symmetry breaking. With increasing temperature and/or baryon density, the QCD vacuum undergoes a phase transition to a deconfined and chiral symmetry restored phase. Besides the ongoing experiments, there are several theoretical tools such as lattice calculations [1,2], various effective models $[3,4]$, AdS/QCD correspondence [5], and the functional renormalization-group method $[6,7]$ used to study the phase diagram of QCD. Lattice results conclusively demonstrated that the phase transition at a vanishing baryon chemical potential is a crossover. The order parameter of chiral symmetry breaking is a quark-antiquark condensate

\footnotetext{
ritesh.ghosh@saha.ac.in

†bithika.karmakar@saha.ac.in

*munshigolam.mustafa@saha.ac.in
}

Published by the American Physical Society under the terms of the Creative Commons Attribution 4.0 International license. Further distribution of this work must maintain attribution to the author(s) and the published article's title, journal citation, and DOI. Funded by SCOAP ${ }^{3}$. which vanishes above the critical temperature in the chiral limit. Chiral susceptibility is the measure of fluctuation of the order parameter. It estimates the response of the chiral condensate with the variation of current quark mass. Measurement of fluctuations is an essential tool used to investigate the properties of QCD matter at extreme conditions e.g., electric charge fluctuation and quark number susceptibility can give insight to the degrees of freedom of the system. Chiral susceptibility has been studied in the framework of lattice QCD [8-12], hard thermal loop approximation [13], chiral perturbation theory [14], NJL model $[15,16]$, the Dyson-Schwinger equation [17], and so on.

Besides, production of a magnetic field in noncentral heavy ion collisions has added a new dimension to the understanding of QCD matter. This extremely strong magnetic field is created by the spectator particles in a direction perpendicular to the reaction plane. This magnetic field can have detectable consequences like chiral magnetic effect (CME) $[18,19]$. Other influences of the magnetic field on the QCD matter viz. change in equation of state (EOS) [20-23], modification in the transport properties [24-30], dilepton production rate [31-35], heavy quark potential [36,37], and damping rate of photons [38] have been studied by different groups of the heavy ion collision community. The magnetic field can also affect the dynamical chiral symmetry breaking.

Some studies suggest that the chiral condensate increases in the presence of a magnetic field. It is argued that in case of a neutral spin-zero pair of a fermion and antifermion, magnetic moments of both point along the same direction. As a result, both magnetic moments can align along the magnetic field direction without creating any frustration in the fermion-antifermion pair [39]. This effect is linked 
to the increase in the phase transition temperature which is known as magnetic catalysis. However, several lattice studies [40] have found the opposite nature i.e., the decrease in phase transition temperature at least for small magnetic fields. This effect has been named as inverse magnetic catalysis. Also these studies revealed that the change in the chiral condensate strongly depends on the temperature and the quark mass. Recently, the chiral susceptibility was calculated using the NJL model in Ref. [41] in the presence of chiral chemical potential and nonzero magnetic field. The magnetic field breaks the flavor symmetry. Hence two distinct peaks of chiral susceptibility for $u$ and $d$ quarks have been observed at large magnetic field.

The strong magnetic field produced in heavy ion collision sharply decays with time $[42,43]$. However, some studies [31,32] have shown that the presence of finite conductivity can make the strong magnetic field survive for a long time. The QCD matter cools down after the collision and undergoes the chiral phase transition at a temperature around $160 \mathrm{MeV}$. In this region the effect of a weak magnetic field is particularly important. In this paper, we consider the magnetic field to be small and use the scale hierarchy $\sqrt{\left|q_{f} B\right|}<g T<T$. In Ref. [13] the chiral susceptibility was computed with zero chemical potential within the hard thermal loop (HTL) approximation. In this paper we, considering a recently obtained effective quark propagator in the presence of a weak magnetic field [44], determine the chiral susceptibility with finite chemical potential in the QCD medium using HTL approximation.

The paper is organized as follows. In Sec. II we describe the static chiral susceptibility. We obtain the general structure of fermion self-energy in the presence of a weak magnetic field and compute the effective propagator in Sec. III. The free chiral susceptibility is calculated in Sec. IV. We compute the HTL chiral susceptibility within a weak magnetic field approximation in Sec. V. The results are described in Sec. VI and we summarize in Sec. VII.

\section{DEFINITION}

The chiral condensate is defined as

$$
\langle\bar{q} q\rangle=\frac{\operatorname{Tr}\left[\bar{q} q e^{-\beta \mathrm{H}}\right]}{\operatorname{Tr}\left[e^{-\beta \mathrm{H}}\right]}=\frac{\partial \Omega}{\partial m_{f}},
$$

where $\mathrm{H}$ is the Hamiltonian of the system. $\Omega=-\frac{T}{V} \ln Z$ is the thermodynamic potential where $\mathrm{Z}$ is the partition function of a quark-antiquark gas. The quark condensate also can be written using a quark propagator as

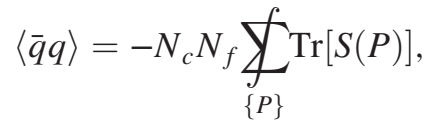

where $N_{c}$ and $N_{f}$ are the numbers of quark colors and flavors respectively. Susceptibility is the measure of the response of a system to a small external force. Chiral susceptibility measures the response of the chiral condensate to the infinitesimal change of the current quark mass $m_{f}$ as

$$
\chi_{c}=-\left.\frac{\partial\langle\bar{q} q\rangle}{\partial m_{f}}\right|_{m_{f}=0} .
$$

\section{GENERAL STRUCTURE OF FERMIONIC TWO-POINT FUNCTION}

Recently the covariant structure of fermion self-energy has been constructed in the presence of temperature and a magnetic field in Ref. [44]. The general covariant structure of fermion self-energy in a weak thermomagnetic field can be written as

$$
\Sigma(P)=-a \not P-b \not h-c^{\prime} \gamma_{5} \not h-d^{\prime} \gamma_{5} \not h,
$$

where $u_{\mu}$ is the four velocity of fluid. The direction of magnetic field $n_{\mu}$ can be written in terms of electromagnetic field tensor $F^{\mu \nu}$ or its dual $\tilde{F}^{\mu \nu}$ and fluid velocity $u_{\mu}$ as

$$
n_{\mu} \equiv \frac{1}{2 B} \epsilon_{\mu \nu \rho \lambda} u^{\nu} F^{\rho \lambda}=\frac{1}{B} u^{\nu} \tilde{F}_{\mu \nu} .
$$

For simplicity we have chosen the fluid rest frame and the magnetic field along the $z$ direction as

$$
\begin{aligned}
& u^{\mu}=(1,0,0,0), \\
& n_{\mu}=(0,0,0,1) .
\end{aligned}
$$

The self-energy structure functions $a, b, c^{\prime}$, and $d^{\prime}$ in Eq. (4) can be calculated using Eqs. (A1), (A2), (A3), and (A4). The structure functions in the presence of a weak magnetic field are calculated up to $\mathcal{O}\left(q_{f} B\right)$ for zero quark chemical potential in Ref. [44]. The calculations are generalized for finite quark chemical potential in Ref. [21]. Here, we compute the structure functions up to $\mathcal{O}\left(q_{f} B\right)^{2}$ in the presence of a chemical potential in Appendix A.

Following the Dyson-Schwinger equation, the effective inverse propagator of a massive fermion can be written as

$$
S_{\text {eff }}^{-1}=\not P-m_{f} \mathbb{I}-\Sigma .
$$

Using Eq. (4) the structure of the inverse propagator of a massive fermion in a thermomagnetic medium can be written as

$$
S_{\text {eff }}^{-1}(P)=(1+a) \not P+b \not h+c^{\prime} \gamma_{5} \not h+d^{\prime} \gamma_{5} \not h-m_{f} \mathbb{I} .
$$

To compute the chiral susceptibility in the presence of a weak magnetic field one requires the effective fermion 
propagator as given in Eqs. (2) and (3). So we need to invert Eq. (9) to get the effective fermion propagator. For a massless case, it is very easy to invert the effective inverse propagator to obtain the general structure of the effective propagator in terms of $\not P, \not l, \gamma_{5} \not h$, and $\gamma_{5} \not h$. To get the structure of the effective propagator in the massive case involving the Dirac matrices, $\not P, \not h, \gamma_{5} \not h, \gamma_{5} \not h$. and $\mathbb{I}$, we adopt the following trick used in Ref. [45].

Let us assume that we need to find the inverse of a matrix $M$. Now we need to choose a matrix $R$ and multiply it with $M$ to get a matrix $U$ as

$$
U=M R .
$$

Now we can write the inverse of the matrix $M$ as

$$
M^{-1}=R U^{-1} .
$$

In our case we need to find the inverse of the matrix $S_{\text {eff }}^{-1}$. Now it is essential to choose $R$ in such a way that we get $U$ in Eq. (10) in a very simple form. Then it would be easy to invert the matrix $U$ and to find the inverse of our desired matrix $S_{\text {eff }}^{-1}$.

Thus we choose $R$ as

$$
R=(1+a) \not p+b \not h-c^{\prime} \gamma_{5} \not l-d^{\prime} \gamma_{5} \not h-m_{f} \mathbb{I} .
$$

From Eqs. (9) and (10), we have

$$
U=S_{\text {eff }}^{-1} R=\alpha \not P+\beta \not h+\delta \gamma_{5}+\lambda \mathbb{I},
$$

where

$$
\begin{aligned}
& \alpha=-2(1+a) m_{f}, \\
& \beta=-2 b m_{f}, \\
& \delta=2\left((1+a) c^{\prime} p_{0}+b c^{\prime}+(1+a) d^{\prime} p_{3}\right), \\
& \lambda=(1+a)^{2} P^{2}+b^{2}+c^{\prime 2}-d^{\prime 2}+m_{f}^{2}+2(1+a) b p_{0} .
\end{aligned}
$$

We can now easily invert the matrix $U$ to get

$$
U^{-1}=\frac{1}{N^{2}}\left(\alpha \not P+\beta \not h+\delta \gamma_{5}-\lambda \mathbb{I}\right),
$$

where

$$
N^{2}=\alpha^{2} P^{2}+2 \alpha \beta p_{0}+\beta^{2}+\delta^{2}-\lambda^{2} .
$$

Following Eq. (11), we get the effective fermion propagator $S_{\text {eff }}$ as

$$
\begin{aligned}
S_{\mathrm{eff}}= & R U^{-1}=\left((1+a) \not P+b \not h-c^{\prime} \gamma_{5} \not h-d^{\prime} \gamma_{5} \not h-m_{f} \mathbb{I}\right) \\
& \times \frac{\alpha \not P+\beta \not h+\delta \gamma_{5}-\lambda \mathbb{I}}{\alpha^{2} P^{2}+2 \alpha \beta p_{0}+\beta^{2}+\delta^{2}-\lambda^{2}} .
\end{aligned}
$$

The dispersion relation for a massive fermion in a weakly magnetized thermal medium can be obtained from the denominator of the effective propagator by setting it to zero.

\section{CHIRAL SUSCEPTIBILITY FOR FREE FERMION IN THE PRESENCE OF A WEAK MAGNETIC FIELD}

We consider a weakly magnetized QCD medium. In the weak magnetic field limit, we work with the scale hierarchy, $\sqrt{\left|q_{f} B\right|}<m_{\text {th }} \sim g T<T$. Now treating $q_{f} B$ as perturbation, the Schwinger propagator for a fermion in the presence of a weak magnetic field can be expanded and written up to $\mathcal{O}\left[\left(q_{f} B\right)^{2}\right]$ as [46]

$$
\begin{aligned}
S(K)= & \frac{K+m_{f}}{K^{2}-m_{f}^{2}}+i \gamma^{1} \gamma^{2} \frac{K_{\|}+m_{f}}{\left(K^{2}-m_{f}^{2}\right)^{2}}\left(q_{f} B\right) \\
& +2\left[\frac{\{(K \cdot u) \not h-(K \cdot n) \not h\}-\not K}{\left(K^{2}-m_{f}^{2}\right)^{3}}-\frac{k_{\perp}^{2}\left(K+m_{f}\right)}{\left(K^{2}-m_{f}^{2}\right)^{4}}\right] \\
& \times\left(q_{f} B\right)^{2}+\mathcal{O}\left[\left(q_{f} B\right)^{3}\right] \\
= & S_{0}+S_{1}+S_{2}+\mathcal{O}\left[\left(q_{f} B\right)^{3}\right] .
\end{aligned}
$$

We can write the chiral condensate for a free fermion in a weak magnetic field up to $\mathcal{O}\left[\left(q_{f} B\right)^{2}\right]$ from Eq. (18) as

$$
\begin{aligned}
& \langle\bar{q} q\rangle_{f}=-N_{c} N_{f} \sum_{\{P\}} \operatorname{Tr}\left[S_{0}(P)+S_{1}(P)+S_{2}(P)\right],
\end{aligned}
$$

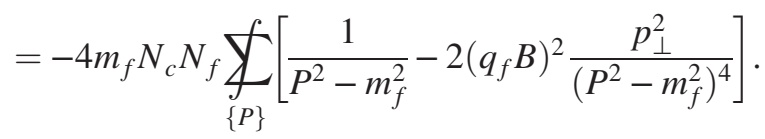

Using the definition in Eq. (3) the chiral susceptibility for a free fermion in a weak magnetic field can be calculated as

$$
\begin{aligned}
& \chi_{c}=-\left.\frac{\partial\langle\bar{q} q\rangle}{\partial m_{f}}\right|_{m_{f}=0}=4 N_{c} N_{f} \bigvee_{\{P\}}\left[\frac{1}{P^{2}}-2\left(q_{f} B\right)^{2} \frac{p_{\perp}^{2}}{\left(P^{2}\right)^{4}}\right] \\
& =\frac{N_{c} N_{f}}{6} T^{2}\left[1+12 \hat{\mu}^{2}-\left(q_{f} B\right)^{2} \frac{\lambda(z)}{16 \pi^{4} T^{4}}\right] \text {, }
\end{aligned}
$$

where $\hat{\mu}=\mu / 2 \pi T$. $\mu$ is the quark chemical potential. The sum integrals are calculated in Appendix B and $\lambda(z)$ is given in Eq. (B16).

\section{HTL CHIRAL SUSCEPTIBILITY IN THE PRESENCE OF A WEAK MAGNETIC FIELD}

Using the effective quark propagator in Eq. (17) chiral condensate $\langle\bar{q} q\rangle$ takes the form 


$$
\begin{aligned}
& \langle\bar{q} q\rangle=-N_{c} N_{f} \bigotimes_{\{P\}} \operatorname{Tr}\left[S_{\text {eff }}(P)\right] \\
& =4 m_{f} N_{c} N_{f} \underbrace{}_{\{P\}} \frac{(1+a)^{2} P^{2}+2(1+a) b p_{0}+b^{2}+d^{\prime 2}-c^{\prime 2}-m_{f}^{2}}{\alpha^{2} P^{2}+2 \alpha \beta p_{0}+\beta^{2}+\delta^{2}-\lambda^{2}} .
\end{aligned}
$$

Chiral susceptibility in the massless limit can be calculated from Eq. (21) as

$$
\begin{aligned}
\chi_{c} & =-\left.\frac{\partial\langle\bar{q} q\rangle}{\partial m_{f}}\right|_{m_{f}=0} \\
& =-4 N_{c} N_{f} \underbrace{}_{\{P\}} \frac{(1+a)^{2} P^{2}+2(1+a) b p_{0}+b^{2}+d^{\prime 2}-c^{\prime 2}}{4\left[(1+a) c^{\prime} p_{0}+b c^{\prime}+(1+a) d^{\prime} p_{3}\right]^{2}-\left[(1+a)^{2} P^{2}+b^{2}+c^{\prime 2}-d^{\prime 2}+2(1+a) b p_{0}\right]^{2}},
\end{aligned}
$$

where the expressions of various structure functions are obtained in Appendix A. Now we expand the expression in Eq. (22) in the series of coupling constant $g$ and keep up to $\mathcal{O}(g)^{4}$ as

$$
\begin{aligned}
\chi_{c}= & 4 N_{c} N_{f} \bigcup_{\{P\}}\left\{\frac{1}{P^{2}}+2 m_{\mathrm{th}}^{2} \frac{1}{P^{4}}+m_{\mathrm{th}}^{4}\left(\frac{4}{P^{6}}+\frac{1}{p^{2} P^{4}}-\frac{2}{p^{2} P^{4}} \mathcal{T}_{p}+\frac{1}{p^{2} p_{0}^{2} P^{2}} \mathcal{T}_{p}^{2}\right)\right. \\
& -m_{\mathrm{eff}}^{\prime 2}\left(\frac{2}{3 P^{4}}-\frac{2 p_{3}^{2}}{p^{2} P^{4}}-\frac{2}{P^{4}} \mathcal{T}_{p}+\frac{2 p_{3}^{2}}{p^{2} P^{4}} \mathcal{T}_{p}\right) \\
& \left.-m_{\mathrm{eff}}^{4}\left(\frac{4 p_{3}^{2}}{p^{2} P^{6}}+\frac{p_{3}^{2}}{p^{4} P^{4}}-\frac{2 p_{3}^{2}}{p^{4} P^{4}} \mathcal{T}_{p}+\frac{3}{p_{0}^{2} P^{4}} \mathcal{T}_{p}^{2}+\frac{p_{3}^{2}}{p^{4} P^{4}} \mathcal{T}_{p}^{2}-\frac{4 p_{3}^{2}}{p^{2} p_{0}^{2} P^{4}} \mathcal{T}_{p}^{2}\right)\right\},
\end{aligned}
$$

where

$$
\begin{aligned}
m_{\mathrm{th}}^{2} & =\frac{g^{2} C_{F} T^{2}}{8}\left(1+4 \hat{\mu}^{2}\right), \\
m_{\mathrm{eff}}^{\prime 2} & =\frac{g^{2} C_{F}\left(q_{f} B\right)^{2} T}{32 \pi m_{f}^{3}}, \\
m_{\mathrm{eff}}^{2} & =4 g^{2} C_{F} \frac{q_{f} B}{16 \pi^{2}}\left[-\frac{1}{4} \aleph(z)-\frac{\pi T}{2 m_{f}}-\frac{\gamma_{E}}{2}\right],
\end{aligned}
$$

with $\aleph(z)$ defined in Eq. (B15) and $C_{F}=\left(N_{c}^{2}-1\right) / 2 N_{c}$ is the QCD Casimir factor. Using the sum integrals listed in Appendix B we find the expression for the chiral susceptibility as

$$
\begin{aligned}
\chi_{c}= & \frac{N_{c} N_{f}}{6} T^{2}\left[1+12 \hat{\mu}^{2}+\frac{3}{\pi^{2}}\left(\frac{\Lambda}{4 \pi T}\right)^{2 \epsilon}\left(\frac{1}{\epsilon}-\aleph(z)\right) \frac{m_{\mathrm{th}}^{2}}{T^{2}}\right. \\
& \left.+\frac{1}{\pi^{2}}\left(\frac{\Lambda}{4 \pi T}\right)^{2 \epsilon}\left(\frac{1}{\epsilon}+\frac{4}{3}-\aleph(z)\right) \frac{m_{\mathrm{eff}}^{\prime 2}}{T^{2}}+\frac{\lambda(z)}{32 \pi^{4}}\left(\pi^{2}-6\right) \frac{m_{\mathrm{th}}^{4}}{T^{4}}-\frac{\lambda(z)}{24 \pi^{4}}\left(\pi^{2}-6\right) \frac{m_{\mathrm{eff}}^{4}}{T^{4}}\right] .
\end{aligned}
$$

We note that the logarithmic divergence comes from the thermal part. A new divergence appears in the presence of the magnetic field. We renormalize the chiral susceptibility within the $\overline{\mathrm{MS}}$ renormalization scheme using the following counterterm:

$$
\Delta \chi_{c}^{\text {counter }}=-\frac{N_{c} N_{f}}{6 \pi^{2} \epsilon}\left(3 m_{\mathrm{th}}^{2}+m_{\mathrm{eff}}^{\prime 2}\right) .
$$

The renormalized chiral susceptibility is given as

$$
\begin{aligned}
\chi_{c}= & \frac{N_{c} N_{f}}{6} T^{2}\left[1+12 \hat{\mu}^{2}+\frac{3}{\pi^{2}}(2 \ln \hat{\Lambda}-2 \ln 2-\aleph(z)) \frac{m_{\mathrm{th}}^{2}}{T^{2}}\right. \\
& \left.+\frac{1}{3 \pi^{2}}(4-3 \aleph(z)+6 \ln \hat{\Lambda}-6 \ln 2) \frac{m_{\mathrm{eff}}^{\prime 2}}{T^{2}}+\frac{\lambda(z)}{32 \pi^{4}}\left(\pi^{2}-6\right) \frac{m_{\mathrm{th}}^{4}}{T^{4}}-\frac{\lambda(z)}{24 \pi^{4}}\left(\pi^{2}-6\right) \frac{m_{\mathrm{eff}}^{4}}{T^{4}}\right],
\end{aligned}
$$




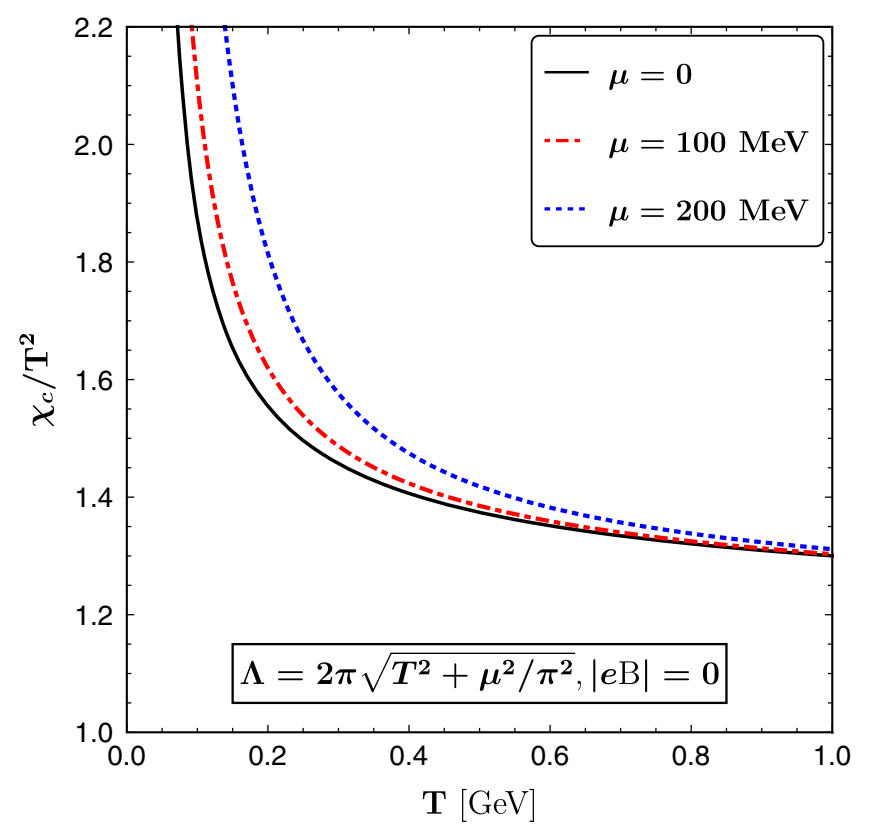

FIG. 1. Variation of chiral susceptibility scaled with $T^{2}$ as a function of temperature for chemical potential 0,100 , and $200 \mathrm{MeV}$ with zero magnetic field.

with $\hat{\Lambda}=\Lambda / 2 \pi T$ and $\hat{\mu}=\mu / 2 \pi T$. The obtained result is completely analytic in the presence of a chemical potential and a weak magnetic field. Here we note that Eq. (27) consists of $\mathcal{O}\left[\left(q_{f} B\right)^{0}\right]$ and $\mathcal{O}\left[\left(q_{f} B\right)^{2}\right]$ terms. The $\mathcal{O}\left[\left(q_{f} B\right)^{0}\right]$ reproduces the thermal chiral susceptibility without the chemical potential obtained in Ref. [13]. The $\mathcal{O}\left[\left(q_{f} B\right)^{2}\right]$ terms are the thermomagnetic correction to the thermal chiral susceptibility.

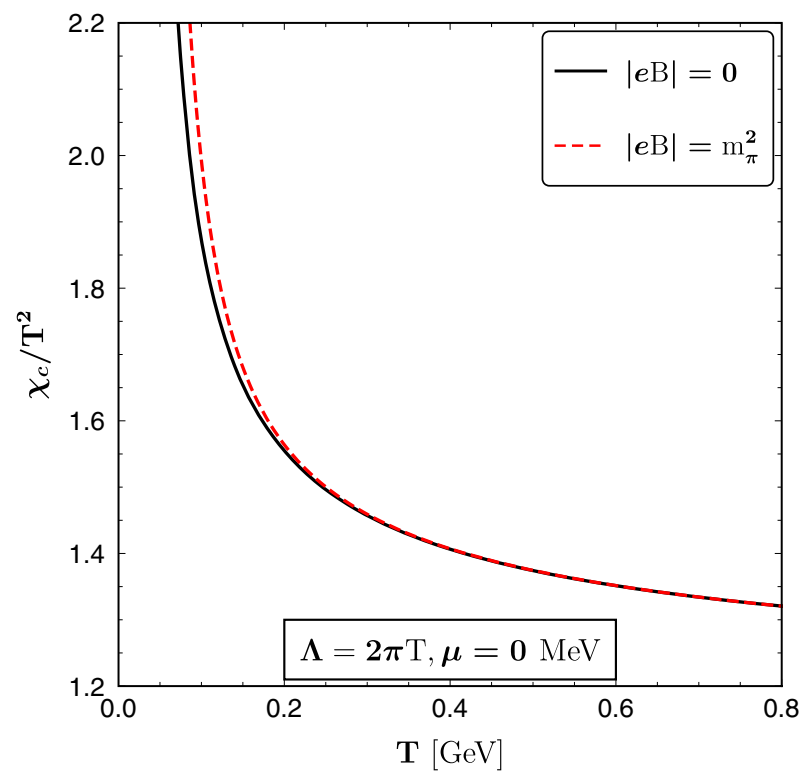

\section{RESULTS}

We consider a magnetic field dependent running coupling [47] as

$$
\alpha_{s}\left(\Lambda^{2},|e B|\right)=\frac{\alpha_{s}\left(\Lambda^{2}\right)}{1+b_{1} \alpha_{s}\left(\Lambda^{2}\right) \ln \left(\frac{\Lambda^{2}}{\Lambda^{2}+|e B|}\right)},
$$

where the one-loop running coupling at renormalization scale reads as

$$
\alpha_{s}\left(\Lambda^{2}\right)=\frac{1}{b_{1} \ln \left(\Lambda^{2} / \Lambda_{\overline{\mathrm{MS}}}^{2}\right)},
$$

with $b_{1}=\frac{11 N_{c}-2 N_{f}}{12 \pi}, \Lambda_{\overline{\mathrm{MS}}}=204 \mathrm{MeV}$ requiring $\alpha_{s}=0.326$ at $1.5 \mathrm{GeV}$ [48]. We choose the renormalization scale as $\Lambda=2 \pi \sqrt{T^{2}+\mu^{2} / \pi^{2}}$. The following results are shown considering two light quark flavors $u$ and $d$.

In Fig. 1 the chiral susceptibility scaled with temperature squared is plotted with temperature in the absence of a magnetic field for zero and nonzero quark chemical potential. The effect of the quark chemical potential is prominent in the low temperature region as can be seen from the figure. A similar plot for a thermal QCD medium and zero chemical potential was obtained in Ref. [13]. For low temperature the chiral susceptibility increases rapidly for both zero and nonzero chemical potential. Here we note that the increase of the chiral susceptibility in the low temperature region does not indicate the chiral phase transition. It is due to the temperature dependence of the coupling constant and for the choice of the renormalization scale [13]. At very high temperature the chiral susceptibility reaches the free value asymptotically.

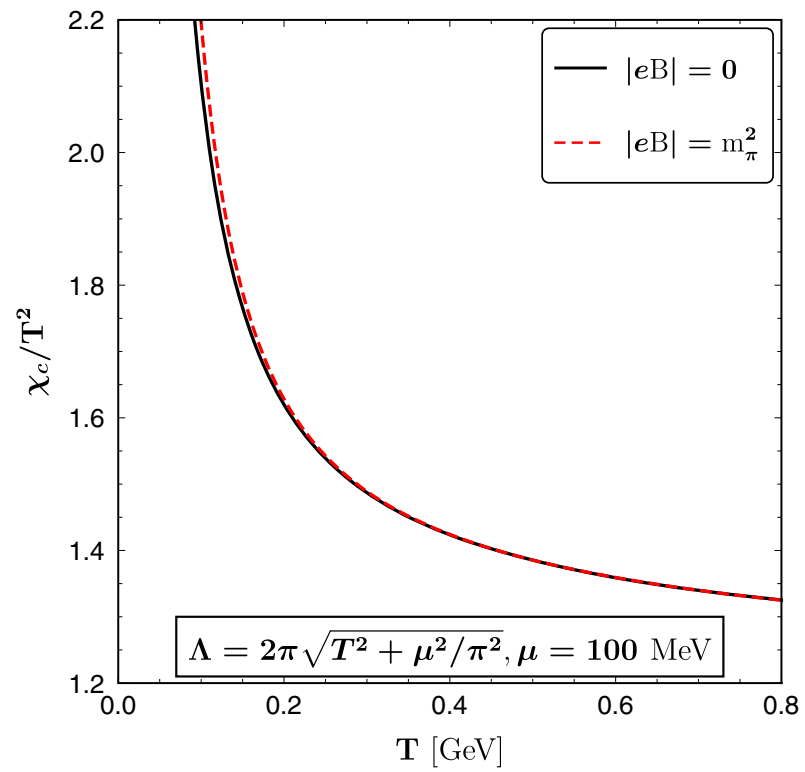

FIG. 2. Variation of chiral susceptibility scaled with $T^{2}$ as a function of $T$ for magnetic field strength $|e B|=0, m_{\pi}^{2}$ with $\mu=0 \mathrm{MeV}$ (left) and $\mu=100 \mathrm{MeV}$ (right). 


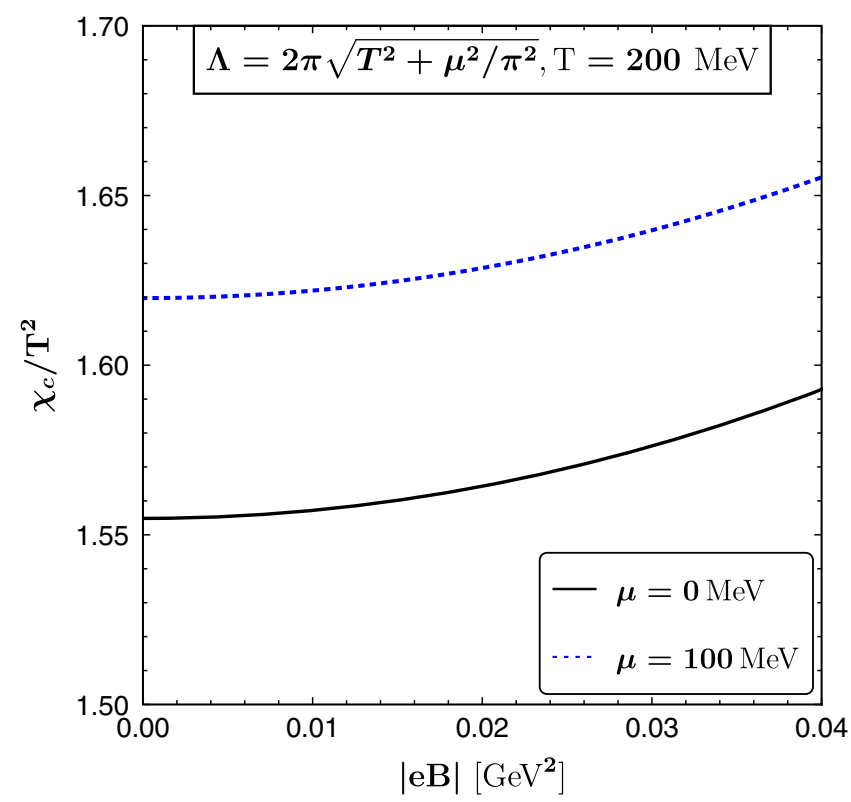

FIG. 3. Scaled chiral susceptibility is plotted as a function of magnetic field strength $|e B|$ for temperature $T=0.2 \mathrm{GeV}$ and $\mu=0 \mathrm{MeV}$ and $100 \mathrm{MeV}$.

The variation of chiral susceptibility scaled with temperature squared for a zero and finite magnetic field is plotted with temperature in Fig. 2. In the left panel of Fig. 2 we have shown the effect of a weak magnetic field on the chiral susceptibility for a zero quark chemical potential, whereas, the same for finite quark chemical potential is shown in the right panel. In the presence of a magnetic field, chiral susceptibility is slightly increased than that of thermal medium in the low temperature region. Since we are

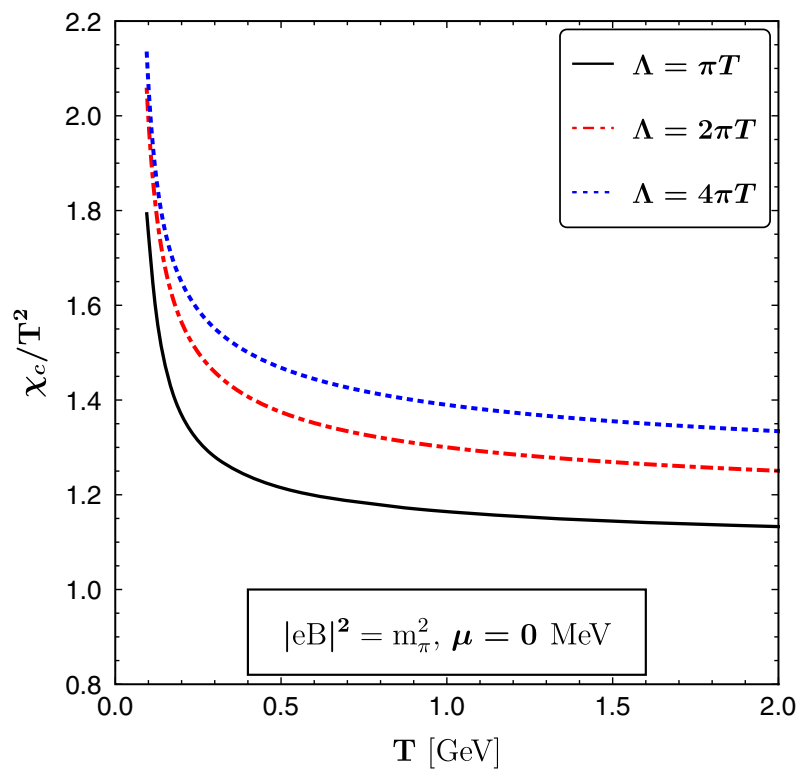

working in a weak magnetic field limit, the increase in susceptibility due to the magnetic field is small. As temperature increases the effect of the magnetic field reduces as temperature becomes the dominant scale.

It should be noted that the scale hierarchy of a weakly magnetized medium is $\sqrt{\left|q_{f} B\right|}<g T<T$. This particular condition is satisfied for around $T>0.14 \mathrm{GeV}$ as we have considered $|e B|=m_{\pi}^{2}=0.14^{2} \mathrm{GeV}^{2}$ in Fig. 2. Thus the weak field and HTL approximations are valid at high temperature. This is consistent with our study because we calculate the chiral susceptibility of the medium in a perturbative region.

The effect of the magnetic field can be seen clearly from Fig. 3 where the variation of the scaled chiral susceptibility is shown with a magnetic field for fixed temperature $T=200 \mathrm{MeV}$. Here we notice the slow increase in the chiral susceptibility with increasing magnetic field for both with and without chemical potential. Here we note that the weak field approximation is valid in this case because $\sqrt{\left|q_{f} B\right|}<T$ is satisfied.

In Fig. 4 the sensitivity of the chiral susceptibility with renormalization scale is shown in the presence of a constant weak magnetic field. Here chiral susceptibility scaled with $T^{2}$ is plotted with temperature for zero (left panel) and finite (right panel) chemical potential by varying renormalization scale $\Lambda$ by factor 2 around its central value $2 \pi \sqrt{T^{2}+\mu^{2} / \pi^{2}}$.

Here we note that HTL approximation is valid above the phase transition temperature where the scale hierarchy $\sqrt{\left|q_{f} B\right|}<g T<T$ is maintained. We have shown the plots of chiral susceptibilities at low temperature just to show the steep increase in the plots.

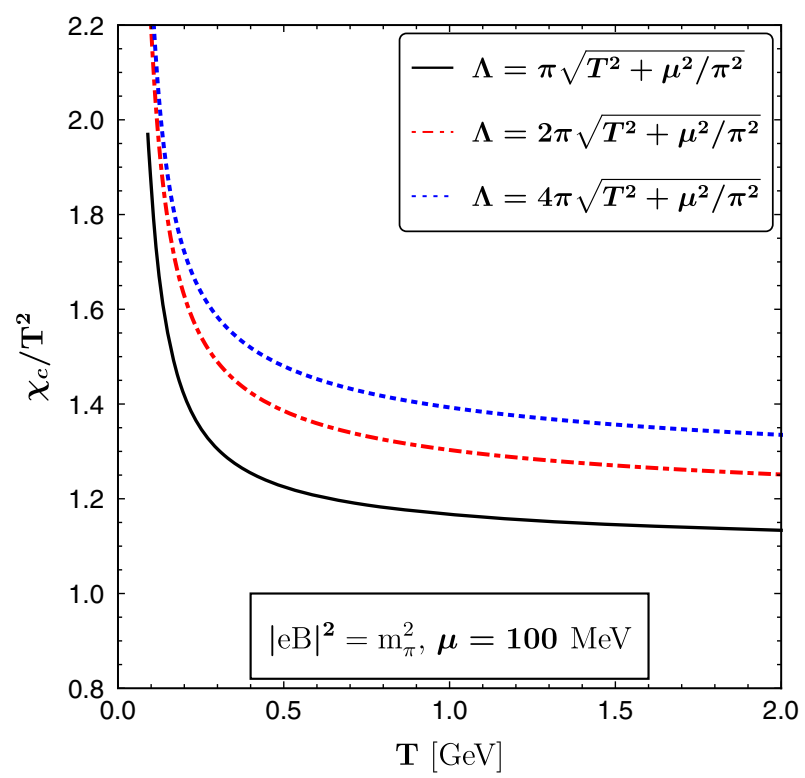

FIG. 4. Variation of chiral susceptibility scaled with $T^{2}$ as a function of temperature for magnetic field strength $m_{\pi}^{2}$ for different values of renormalization scale. 


\section{SUMMARY}

We have investigated the effect of a magnetic field on the chiral susceptibility of quark-gluon plasma within HTL approximation in the presence of a finite chemical potential. The general structure of an effective massive fermion propagator is constructed for a thermomagnetic medium. Then the self-energy structure functions up to $\mathcal{O}\left[\left(q_{f} B\right)^{2}\right]$ in the presence of a chemical potential have been calculated in the weak magnetic field regime using the scale hierarchy $\sqrt{\left|q_{f} B\right|}<g T<T$. The quark condensate is computed using an effective quark propagator in the presence of a magnetic field. Finally we obtain a completely analytic expression for chiral susceptibility in a hot and dense weakly magnetized QCD medium. We have subtracted the UV divergence via the $\overline{\mathrm{MS}}$ renormalization scheme. It is found that the chiral susceptibility is increased due to the presence of the chemical potential as well as the background magnetic field. At high temperature the effect of a magnetic field on chiral susceptibility becomes feeble.

\section{ACKNOWLEDGMENTS}

R. G. is funded by University Grants Commission (UGC). B. K. and M. G. M. are funded by the Department of Atomic Energy (DAE), India, via the project TPAES.

\section{APPENDIX A: STRUCTURE FUNCTIONS}

The general form of the various structure functions can be written from Eq. (4) as

$$
\begin{gathered}
a=\frac{1}{4} \frac{\operatorname{Tr}(\Sigma \not P)-(P \cdot u) \operatorname{Tr}(\Sigma \not \iota)}{(P \cdot u)^{2}-P^{2}}, \\
b=\frac{1}{4} \frac{-(P \cdot u) \operatorname{Tr}(\Sigma \not \supset)+P^{2} \operatorname{Tr}(\Sigma \not \ell)}{(P \cdot u)^{2}-P^{2}}, \\
c^{\prime}=-\frac{1}{4} \operatorname{Tr}\left(\not h \Sigma \gamma_{5}\right), \\
d^{\prime}=\frac{1}{4} \operatorname{Tr}\left(\not h \Sigma \gamma_{5}\right) .
\end{gathered}
$$

The structure functions in the presence of a magnetic field depends on three Lorentz scalars:

$$
\begin{gathered}
p_{0}=P^{\mu} u_{\mu}, \\
p_{3}=P^{\mu} n_{\mu}=p_{z}, \\
p_{\perp}=\left[-\left(P^{\mu} P_{\mu}\right)^{2}+\left(P^{\mu} u_{\mu}\right)^{2}-\left(P^{\mu} n_{\mu}\right)^{2}\right]^{1 / 2}=\left(p_{1}^{2}+p_{2}^{2}\right)^{1 / 2} .
\end{gathered}
$$

A free quark propagator in a weak magnetic field is given in Eq. (18). Now the one-loop quark self-energy up to $\mathcal{O}\left[\left(q_{f} B\right)^{2}\right]$ can be written as

$$
\begin{aligned}
\Sigma(P) & =g^{2} C_{F} \int_{\{K\}} \gamma_{\mu}\left(S_{0}(K)+S_{1}(K)+S_{2}(K)\right) \gamma^{\mu} \frac{1}{(K-P)^{2}} \\
& =\Sigma_{0}+\Sigma_{1}+\Sigma_{2} .
\end{aligned}
$$

From (A1), the structure function $a$ can be written up to $\mathcal{O}\left[\left(q_{f} B\right)^{2}\right]$ as

$$
\begin{aligned}
a= & \frac{1}{4} \frac{\operatorname{Tr}\left(\Sigma_{0} \not P\right)-(P \cdot u) \operatorname{Tr}\left(\Sigma_{0} \not \iota\right)}{(P \cdot u)^{2}-P^{2}} \\
& +\frac{1}{4} \frac{\operatorname{Tr}\left(\Sigma_{2} \not P\right)-(P \cdot u) \operatorname{Tr}\left(\Sigma_{2} \not \iota\right)}{(P \cdot u)^{2}-P^{2}}, \\
= & a_{0}+a_{B},
\end{aligned}
$$

where $a_{0}$ is a purely thermal contribution $(B=0)$ and $a_{B}$ is the magnetic correction of $\mathcal{O}\left[\left(q_{f} B\right)^{2}\right]$ coming from $\Sigma_{2}$. The $\mathcal{O}\left[\left(q_{f} B\right)\right]$ corrections coming from $\Sigma_{1}$ vanish due to the trace of odd number of gamma matrices.

Similarly structure function $b$ can be written as

$$
\begin{aligned}
b= & \frac{1}{4} \frac{-(P \cdot u) \operatorname{Tr}\left(\Sigma_{0} \not P\right)+P^{2} \operatorname{Tr}\left(\Sigma_{0} \not \iota\right)}{(P \cdot u)^{2}-P^{2}} \\
& +\frac{1}{4} \frac{-(P \cdot u) \operatorname{Tr}\left(\Sigma_{2} \not P\right)+P^{2} \operatorname{Tr}\left(\Sigma_{2} \not \iota\right)}{(P \cdot u)^{2}-P^{2}}, \\
= & b_{0}+b_{B} .
\end{aligned}
$$

The thermal part of the structure functions $a$ and $b$ can be calculated using the quark self-energy diagram in Fig. 5 as [44]

$$
\begin{gathered}
a_{0}\left(p_{0}, p\right)=-\frac{m_{\mathrm{th}}^{2}}{p^{2}} \int \frac{d \Omega}{4 \pi} \frac{p \cdot \hat{k}}{P \cdot \hat{K}}, \\
b_{0}\left(p_{0}, p\right)=\frac{m_{\mathrm{th}}^{2}}{p} \int \frac{d \Omega}{4 \pi} \frac{(P \cdot u)(p \cdot \hat{k})-p^{2}}{P \cdot \hat{K}},
\end{gathered}
$$

where thermal mass is given as

$$
m_{\mathrm{th}}^{2}=\frac{g^{2} C_{F} T^{2}}{8}\left(1+4 \hat{\mu}^{2}\right)
$$

with $\hat{\mu}=\mu / 2 \pi T$ and $C_{F}=\left(N_{c}^{2}-1\right) / 2 N_{c}$.

Now we derive the $\mathcal{O}\left[\left(q_{f} B\right)^{2}\right]$ corrections to the structure functions $a$ and $b$. To get the expression of $a_{B}$ and $b_{B}$ we need to perform the following sum-integrations:

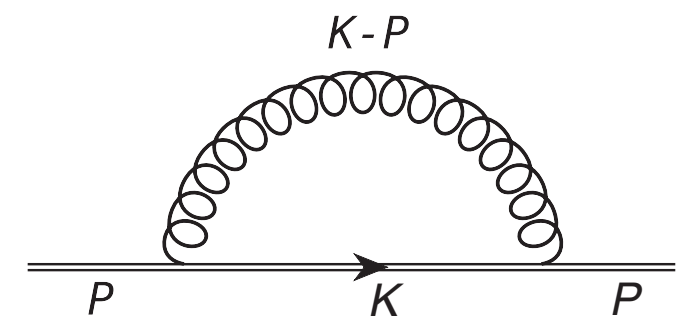

FIG. 5. Self-energy diagram for a quark in the presence of a background magnetic field. The double line indicates the modified quark propagator in the presence of a weak magnetic field. 


$$
\begin{aligned}
\operatorname{Tr}\left(\Sigma_{2} \not l\right)= & 16 g^{2} C_{F}\left(q_{f} B\right)^{2} T \bigvee_{\{K\}} \frac{k_{\perp}^{2} k_{0}}{\left(K^{2}-m_{f}^{2}\right)^{4} Q^{2}} \\
= & \frac{16}{6} g^{2} C_{F}\left(q_{f} B\right)^{2} \int \frac{k^{2} d k d \Omega}{(2 \pi)^{3}} \frac{\partial^{3}}{\partial\left(m_{f}^{2}\right)^{3}}\left[-\frac{k_{\perp}^{2}}{4 \sqrt{k^{2}+m_{f}^{2}}}\left(n_{F}\left(\sqrt{k^{2}+m_{f}^{2}}\right)+n_{B}\left(\sqrt{k^{2}+m_{f}^{2}}\right)\right)\right] \\
& \times\left(\frac{1}{p_{0}-p \cdot \hat{k}}+\frac{1}{p_{0}+p \cdot \hat{k}}\right) \\
= & \frac{g^{2} C_{F}\left(q_{f} B\right)^{2}}{6 \pi^{3} T^{2}} \Gamma(5) \frac{\partial^{3}}{\partial\left(y^{2}\right)^{3}}\left[h_{5}(y)+f_{5}(y)\right] \int d \Omega \frac{\hat{k}_{\perp}^{2}}{P \cdot \hat{K}} \\
= & \frac{g^{2} C_{F} T\left(q_{f} B\right)^{2}}{8 \pi m_{f}^{3}} \int \frac{d \Omega}{4 \pi} \frac{\hat{k}_{\perp}^{2}}{P \cdot \hat{K}}
\end{aligned}
$$

where we have used well-known functions

$$
f_{n+1}(y)=\frac{1}{\Gamma(n+1)} \int_{0}^{\infty} \frac{d x x^{n}}{\sqrt{x^{2}+y^{2}}} n_{F}\left(\sqrt{x^{2}+y^{2}}\right)
$$

and

$h_{n+1}(y)=\frac{1}{\Gamma(n+1)} \int_{0}^{\infty} \frac{d x x^{n}}{\sqrt{x^{2}+y^{2}}} n_{B}\left(\sqrt{x^{2}+y^{2}}\right)$,

which satisfy the recursion relations [21]

$$
\frac{\partial f_{n+1}}{\partial y^{2}}=-\frac{f_{n-1}}{2 n}
$$

$$
\frac{\partial h_{n+1}}{\partial y^{2}}=-\frac{h_{n-1}}{2 n} .
$$

Expressions for $f_{1}(y)$ and $h_{1}(y)$ are given as

$$
\begin{aligned}
& f_{1}(y)=\frac{\pi}{2 y}+\frac{1}{2} \ln \left(\frac{y}{4 \pi}\right)+\cdots, \\
& h_{1}(y)=-\frac{1}{2} \ln \left(\frac{y}{4 \pi}\right)+\frac{1}{4} \aleph(z)+\cdots,
\end{aligned}
$$

where $\boldsymbol{\aleph}(z)$ is defined in Eq. (B12).

Now we compute the following trace as

$$
\operatorname{Tr}\left(\Sigma_{2} \not P\right)=X_{1}+X_{2}+X_{3}+X_{4}
$$

where

$$
\begin{aligned}
X_{1}= & -16 g^{2} C_{F}\left(q_{f} B\right)^{2} \bigcup_{\{K\}} \frac{k(p \cdot \hat{k})}{\left(K^{2}-m_{f}^{2}\right)^{3} Q^{2}} \\
= & -8 g^{2} C_{F}\left(q_{f} B\right)^{2} \int \frac{k^{2} d k d \Omega}{(2 \pi)^{3}} \frac{\partial^{2}}{\partial\left(m_{f}^{2}\right)^{2}}\left[\frac{k(p \cdot \hat{k})}{4 q \sqrt{k^{2}+m_{f}^{2}}}\left(n_{F}\left(\sqrt{k^{2}+m_{f}^{2}}\right)+n_{B}\left(\sqrt{k^{2}+m_{f}^{2}}\right)\right)\right] \\
& \times\left(\frac{1}{p_{0}+p \cdot \hat{k}}-\frac{1}{p_{0}-p \cdot \hat{k}}\right) \\
= & -\frac{g^{2} C_{F}\left(q_{f} B\right)^{2}}{8 \pi^{3} T^{2}} \frac{\partial}{\partial y^{2}}\left[h_{1}(y)+f_{1}(y)\right] \int d \Omega \frac{p \cdot \hat{k}}{P \cdot \hat{K}} \\
= & \frac{g^{2} C_{F} T\left(q_{f} B\right)^{2}}{8 \pi m_{f}^{3}} \int \frac{d \Omega}{4 \pi} \frac{p \cdot \hat{k}}{P \cdot \hat{K}} .
\end{aligned}
$$


By performing similar calculations we get

$$
\begin{aligned}
X_{2} & =16 g^{2} C_{F}\left(q_{f} B\right)^{2} \bigcup_{\{K\}} \frac{k \hat{k}_{3} p_{3}}{\left(K^{2}-m_{f}^{2}\right)^{3} Q^{2}} \\
& =-\frac{g^{2} C_{F} T\left(q_{f} B\right)^{2}}{8 \pi m_{f}^{3}} \int \frac{d \Omega}{4 \pi} \frac{p_{3} \hat{k}_{3}}{P \cdot \hat{K}}, \\
X_{3} & =16 g^{2} C_{F}\left(q_{f} B\right)^{2} \bigcup_{\{K\}} \frac{k_{\perp}^{2} k_{0} p_{0}}{\left(K^{2}-m_{f}^{2}\right)^{4} Q^{2}} \\
& =\frac{g^{2} C_{F} T\left(q_{f} B\right)^{2}}{8 \pi m_{f}^{3}} \int \frac{d \Omega}{4 \pi} \frac{p_{0} \hat{k}_{\perp}^{2}}{P \cdot \hat{K}}, \\
X_{4} & =-16 g^{2} C_{F}\left(q_{f} B\right)^{2} \bigcup_{\{K\}} \frac{k_{\perp}^{2} k(p \cdot \hat{k})}{\left(K^{2}-m_{f}^{2}\right)^{4} Q^{2}} \\
& =-\frac{g^{2} C_{F} T\left(q_{f} B\right)^{2}}{8 \pi m_{f}^{3}} \int \frac{d \Omega}{4 \pi} \frac{\hat{k}_{\perp}^{2}(p \cdot \hat{k})}{P \cdot \hat{K}} .
\end{aligned}
$$

So we obtain the expression of $a_{B}$ and $b_{B}$ as

$$
\begin{aligned}
a_{B} & =\frac{g^{2} C_{F} T\left(q_{f} B\right)^{2}}{32 \pi m_{f}^{3}} \frac{1}{p^{2}} \int \frac{d \Omega}{4 \pi} \frac{\hat{k}_{3}^{2}(p \cdot \hat{k})-p_{3} \hat{k}_{3}}{P \cdot \hat{K}}, \\
b_{B} & =\frac{g^{2} C_{F} T\left(q_{f} B\right)^{2}}{32 \pi m_{f}^{3}} \frac{1}{p} \int \frac{d \Omega}{4 \pi} \frac{\hat{k}_{3}(P \cdot u)\left(\hat{p}_{3}-\hat{p} \cdot \hat{k} \hat{k}_{3}\right)-p \hat{k}_{\perp}^{2}}{P \cdot \hat{K}} .
\end{aligned}
$$

Two other structure functions are given as [44]

$$
\begin{aligned}
c^{\prime} & =-m_{\mathrm{eff}}^{2} \int \frac{d \Omega}{4 \pi} \frac{\hat{K} \cdot n}{P \cdot \hat{K}}, \\
d^{\prime} & =m_{\mathrm{eff}}^{2} \int \frac{d \Omega}{4 \pi} \frac{\hat{K} \cdot u}{P \cdot \hat{K}},
\end{aligned}
$$

where the contribution comes only from the $\Sigma_{1}$ term. The contributions from $\Sigma_{0}$ and $\Sigma_{2}$ vanish due the trace of odd number of gamma matrices.

Here we note that

$$
m_{\mathrm{eff}}^{2}=4 g^{2} C_{F} M_{f}^{2}\left(T, m_{f}, q_{f} B\right),
$$

where the thermomagnetic mass for flavor $f$ is given as

$$
M_{f}^{2}\left(T, m_{f}, q_{f} B\right)=\frac{q_{f} B}{16 \pi^{2}}\left[-\frac{1}{4} \aleph(z)-\frac{\pi T}{2 m_{f}}-\frac{\gamma_{E}}{2}\right],
$$

where $\boldsymbol{\aleph}(z)$ is defined in Eq. (B15).

We rearrange the inverse of effective propagator in different way,

$$
\begin{aligned}
S_{\text {eff }}^{-1}(P) & =(1+a) \not P+b \not h+c^{\prime} \gamma_{5} \not h+d^{\prime} \gamma_{5} \not h-m_{f} \mathbb{I} \\
& =c p_{0} \gamma_{0}-d p_{i} \gamma_{i}+c^{\prime} \gamma_{5} \not h+d^{\prime} \gamma_{5} \not h-m_{f} \mathbb{I},
\end{aligned}
$$

where

$$
\begin{aligned}
& c=1+\left(a_{0}+\frac{b_{0}}{p_{0}}\right)+\left(a_{B}+\frac{b_{B}}{p_{0}}\right)=1-a_{0}^{\prime}-a_{B}^{\prime}, \\
& d=1+a_{0}+a_{B},
\end{aligned}
$$

with

$$
\begin{aligned}
a_{0}= & \frac{m_{\mathrm{th}}^{2}}{p^{2}}\left(1-\mathcal{T}_{p}\right), \\
a_{0}^{\prime}= & \frac{m_{\mathrm{th}}^{2}}{p_{0}^{2}} \mathcal{T}_{p}, \\
a_{B}= & \frac{m_{\mathrm{eff}}^{\prime 2}}{6 p^{2}}\left(1-\frac{3 p_{3}^{2}}{p^{2}}\right)\left(\frac{3 p_{0}^{2}}{p^{2}}\left(1-\mathcal{T}_{p}\right)-2+3 \mathcal{T}_{p}\right), \\
a_{B}^{\prime}= & \frac{m_{\mathrm{eff}}^{\prime 2}}{2 p_{0}^{2}}\left(3 \frac{p_{0}^{2} p_{3}^{2}}{p^{4}}\left(1-\mathcal{T}_{p}\right)+\mathcal{T}_{p}\right. \\
& \left.+\frac{1}{p^{2}}\left(p_{3}^{2} \mathcal{T}_{p}-p_{0}^{2}\left(1-\mathcal{T}_{p}\right)\right)\right), \\
c^{\prime}= & \frac{p_{3} m_{\mathrm{eff}}^{2}}{p^{2}}\left(1-\mathcal{T}_{p}\right), \\
d^{\prime}= & \frac{m_{\mathrm{eff}}^{2}}{p_{0}} \mathcal{T}_{p} .
\end{aligned}
$$

We expressed all the structure functions in terms of

$$
\mathcal{T}_{p}=\int \frac{d \Omega}{4 \pi} \frac{p_{0}}{p_{0}-p \cdot \hat{k}} .
$$

Here we have defined

$$
m_{\mathrm{eff}}^{\prime 2}=\frac{g^{2} C_{F}\left(q_{f} B\right)^{2} T}{32 \pi m_{f}^{3}}
$$

with $C_{F}=\left(N_{c}^{2}-1\right) / 2 N_{c}$. We can see that $m_{\text {eff }}^{\prime 2}$ and $m_{\text {eff }}^{2}$ diverge when the current quark mass vanishes $\left(m_{f} \rightarrow 0\right)$. It is regulated by thermal mass $m_{t h}$ of the fermion as discussed in Refs. [49,50].

\section{APPENDIX B: SUM INTEGRALS}

The dimensionally regularized sum integrals are defined as

$$
{\underset{\{P\}}{ }}_{\{P}=\left(\frac{e^{\gamma_{E}} \Lambda^{2}}{4 \pi}\right)^{\epsilon} T \sum_{p_{0}=(2 n+1) \pi T i+\mu} \int \frac{d^{d-2 \epsilon} p}{(2 \pi)^{d-2 \epsilon}},
$$

where $\Lambda$ can be identified as the $\overline{\mathrm{MS}}$ renormalization scale which also introduces the factor $\left(\frac{e^{\prime} E}{4 \pi}\right)^{\epsilon}$ along with it where $\gamma_{E}$ is the Euler-Mascheroni constant. 
The sum integrals are related by the following equations:

$$
\begin{aligned}
& \searrow_{\{P\}} \frac{1}{P^{4}}=-\frac{d-2}{2} \bigcup_{\{P\}} \frac{1}{p^{2} P^{2}}, \\
& \bigcup_{\{P\}} \frac{1}{p^{2} P^{4}}=-\frac{d-4}{2} \underbrace{}_{\{P\}} \frac{1}{p^{4} P^{2}} .
\end{aligned}
$$

\section{One-loop sum integrals}

We list the fermionic sum integrals as $[13,21]$

$$
\begin{gathered}
\sum_{\{P\}} \frac{1}{P^{2}}=\frac{T^{2}}{24}\left(\frac{\Lambda}{4 \pi T}\right)^{2 \epsilon}\left[1+12 \hat{\mu}^{2}\right], \\
\underbrace{}_{\{P\}} \frac{1}{P^{4}}=\frac{1}{(4 \pi)^{2}}\left(\frac{\Lambda}{4 \pi T}\right)^{2 \epsilon}\left[\frac{1}{\epsilon}-\aleph(z)\right], \\
\underbrace{}_{\{P\}} \frac{p_{3}^{2}}{p^{2} P^{4}}=\frac{1}{3(4 \pi)^{2}}\left(\frac{\Lambda}{4 \pi T}\right)^{2 \epsilon}\left[\frac{1}{\epsilon}+\frac{2}{3}-\aleph(z)\right], \\
\sum_{\{P\}} \frac{1}{P^{6}}=\frac{1}{(2 \pi)^{4}}\left(\frac{\Lambda}{4 \pi T}\right)^{2 \epsilon} \frac{\lambda(z)}{32 T^{2}}, \\
\sum_{\{P\}} \frac{1}{p^{4} P^{2}}=-\frac{1}{(2 \pi)^{4}}\left(\frac{\Lambda}{4 \pi T}\right)^{2 \epsilon} \frac{\lambda(z)}{4 T^{2}}, \\
\sum_{\{P\}} \frac{1}{p^{2} P^{4}}=-\frac{1}{(2 \pi)^{4}}\left(\frac{\Lambda}{4 \pi T}\right)^{2 \epsilon} \frac{\lambda(z)}{8 T^{2}}, \\
\sum_{\{P\}} \frac{p_{3}^{2}}{p^{2} P^{6}}=\frac{1}{(2 \pi)^{4}}\left(\frac{\Lambda}{4 \pi T}\right)^{2 \epsilon} \frac{\lambda(z)}{96 T^{2}}, \\
\sum_{\{P\}} \frac{p_{3}^{2}}{p^{4} P^{4}}=-\frac{1}{(2 \pi)^{4}}\left(\frac{\Lambda}{4 \pi T}\right)^{2 \epsilon} \frac{\lambda(z)}{24 T^{2}} .
\end{gathered}
$$

Here we list the frequently used functions in the sum integrals

$$
\begin{gathered}
\aleph(z) \equiv \Psi(z)+\Psi\left(z^{*}\right), \\
\lambda(z) \equiv \frac{d^{2}}{d z^{2}}\left(\Psi(z)+\Psi\left(z^{*}\right)\right),
\end{gathered}
$$

where $z$ is a general complex number; here $z=1 / 2-i \hat{\mu}$. $\zeta$ and $\Psi$ denote the Riemann zeta function and the digamma function respectively. The digamma function can be written as

$$
\Psi(z) \equiv \frac{\Gamma^{\prime}(z)}{\Gamma(z)} .
$$

We write the functional form of $\aleph(1 / 2-i \hat{\mu})$ and $\lambda(1 / 2-i \hat{\mu})$ for small $\hat{\mu}$ below:

$$
\begin{aligned}
\aleph(z)= & -2 \gamma_{E}-4 \ln 2+14 \zeta(3) \hat{\mu}^{2}-62 \zeta(5) \hat{\mu}^{4} \\
& +254 \zeta(7) \hat{\mu}^{6}+\mathcal{O}\left(\hat{\mu}^{8}\right), \\
\lambda(z)= & -4\left[7 \zeta(3)-186 \zeta(5) \hat{\mu}^{2}+1905 \zeta(7) \hat{\mu}^{4}\right. \\
& \left.-14308 \zeta(9) \hat{\mu}^{6}\right]+\mathcal{O}\left(\hat{\mu}^{8}\right) .
\end{aligned}
$$

\section{One-loop HTL sum integrals used in the magnetic case}

We also need one-loop HTL sum integrals which involve the angular average defined earlier in Eq. (A34). For brevity, henceforth we will use the notation $c=\cos \theta$ for single angular average and $c_{i}=\cos \theta_{i}$ for multiple angular averages. We list the sum integrals below:

$$
\begin{gathered}
\sum_{\{P\}} \frac{1}{P^{4}} \mathcal{T}_{p}=\frac{d-4}{d-5} \bigvee_{\{P\}} \frac{1}{P^{4}}, \\
\sum_{\{P\}} \frac{1}{p^{2} P^{4}} \mathcal{T}_{p}=\left(\Delta_{1}-\frac{d-4}{2} \Delta_{2}\right) \bigcup_{\{P\}} \frac{1}{p^{4} P^{2}}, \\
\sum_{\{P\}} \frac{p_{3}^{2}}{p^{2} P^{4}} \mathcal{T}_{p}=\left(\frac{d-4}{d-2}\left(1+\Delta_{0}\right)+\frac{2}{d-2} \Delta_{3}^{\prime}\right) \Delta_{3} \bigvee_{\{P\}} \frac{1}{P^{4}}, \\
\sum_{\{P\}} \frac{\mathcal{T}_{p}^{2}}{p^{2} p_{0}^{2} P^{2}}=\Delta_{4} \underbrace{}_{\{P\}} \frac{1}{p^{4} P^{2}},
\end{gathered}
$$$$
\sum_{\{P\}} \frac{p_{3}^{2}}{p^{4} P^{4}} \mathcal{T}_{p}=\left(\Delta_{1}-\frac{d-4}{2} \Delta_{2}\right) \Delta_{3} \bigvee_{\{P\}} \frac{1}{p^{4} P^{2}},
$$$$
\bigcup_{\{P\}} \frac{1}{p_{0}^{2} P^{4}} \mathcal{T}_{p}^{2}=\Delta_{5} \bigcup_{\{P\}} \frac{1}{p^{4} P^{2}},
$$$$
\bigcup_{\{P\}} \frac{p_{3}^{2}}{p^{4} P^{4}} \mathcal{T}_{p}^{2}=\Delta_{3} \Delta_{6} \bigcup_{\{P\}} \frac{1}{p^{4} P^{2}},
$$$$
\bigcup_{\{P\}} \frac{p_{3}^{2}}{p^{2} p_{0}^{2} P^{4}} \mathcal{T}_{p}^{2}=\Delta_{3} \Delta_{5} \bigvee_{\{P\}} \frac{1}{p^{4} P^{2}},
$$ 
where $\Delta$ 's are the various angular averages which we list below. The symbol \langle\rangle$_{c}$ in the angular averages depicts the standard definition used in Ref. [51].

$$
\begin{gathered}
\Delta_{0}=\left\langle\frac{c^{2}}{1-c^{2}}\right\rangle_{c}=-\frac{1}{2 \epsilon}+\mathcal{O}[\epsilon]^{3}, \\
\Delta_{1}=\left\langle\frac{c^{6-d}-c^{2}}{\left(1-c^{2}\right)^{2}}\right\rangle_{c}=\frac{1}{4 \epsilon}-\frac{1}{4}+\ln 2+\epsilon\left[-\frac{3}{4}+\frac{\pi^{2}}{6}+(\ln 2)^{2}-2 \ln 2\right]+\mathcal{O}[\epsilon]^{2}, \\
\Delta_{2}=\left\langle\frac{1}{1-c^{2}}\right\rangle_{c}=-\frac{1}{2 \epsilon}+1+\mathcal{O}[\epsilon], \\
\Delta_{3}=\left\langle c^{2}\right\rangle_{c}=\frac{1}{3}+\frac{2 \epsilon}{9}+\mathcal{O}[\epsilon]^{2}, \\
\Delta_{5}=\left\langle\frac{1-c^{4-d}}{\left(1-c^{2}\right)^{2}}\right\rangle_{c}=-\frac{1}{4 \epsilon}+\frac{1}{4}+\frac{3 \epsilon}{4}-\frac{3 \epsilon^{2}}{4}+\mathcal{O}[\epsilon]^{3}, \\
\Delta_{6}=\left\langle\frac{c_{1}^{6-d}-c_{1}^{2}}{\left(c_{1}^{2}-c_{2}^{2}\right)\left(1-c_{1}^{2}\right)^{2}}-\frac{d-4}{2} \frac{1-c_{1}^{6-d}}{\left(c_{1}^{2}-c_{2}^{2}\right)\left(1-c_{1}^{2}\right)}+c_{1} \leftrightarrow c_{2}^{2}\right\rangle_{c_{1}, c_{2}}=\frac{1}{24}\left[6-c_{1}^{2} \leftrightarrow c_{2}^{2}\right\rangle_{c_{1}, c_{2}}=\frac{1}{12}\left[24 \ln 2-\pi^{2}\right]+\mathcal{O}[\epsilon], \\
\left.\left(1-c_{1}^{2}\right)^{2}-\frac{d-4}{2} \frac{1}{\left(c_{1}^{2}-c_{2}^{2}\right)\left(1-c_{1}^{2}\right)}+c_{1} \leftrightarrow c_{2}\right\rangle_{c_{1}, c_{2}}=\frac{1}{8}\left[2-\pi^{2}+16 \ln 2\right]+\mathcal{O}[\epsilon] .
\end{gathered}
$$

Using the expressions of angular averages we obtain the results of HTL sum integrals for a magnetic case as

$$
\begin{gathered}
\sum_{\{P\}} \frac{1}{P^{4}} \mathcal{T}_{p}=\frac{1}{2(4 \pi)^{2}}\left(\frac{\Lambda}{4 \pi T}\right)^{2 \epsilon}\left[\frac{1}{\epsilon}+1-\aleph(z)\right], \\
\underbrace{}_{\{P\}} \frac{1}{p^{2} P^{4}} \mathcal{T}_{p}=-\frac{1}{(2 \pi)^{4}}\left(\frac{\Lambda}{4 \pi T}\right)^{2 \epsilon} \frac{\lambda(z)}{16 T^{2}}(-1+4 \ln 2), \\
\sum_{\{P\}} \frac{p_{3}^{2}}{p^{2} P^{4}} \mathcal{T}_{p}=\frac{1}{6(4 \pi)^{2}}\left(\frac{\Lambda}{4 \pi T}\right)^{2 \epsilon}\left[\frac{1}{\epsilon}+\frac{5}{3}-\aleph(z)\right], \\
\bigcup_{\{P\}} \frac{1}{p^{2} p_{0}^{2} P^{2}} \mathcal{T}_{p}^{2}=\frac{1}{(2 \pi)^{4}}\left(\frac{\Lambda}{4 \pi T}\right)^{2 \epsilon} \frac{\lambda(z)}{48 T^{2}}\left(\pi^{2}-24 \ln 2\right), \\
\underbrace{}_{\{P\}} \frac{p_{3}^{2}}{p^{4} P^{4}} \mathcal{T}_{p}=-\frac{1}{(2 \pi)^{4}}\left(\frac{\Lambda}{4 \pi T}\right)^{2 \epsilon} \frac{\lambda(z)}{48 T^{2}}(-1+4 \ln 2), \\
\sum_{\{P\}}^{\sum_{p_{0}} \frac{1}{p^{2} P^{4}}} \mathcal{T}_{p}^{2}=\frac{1}{(2 \pi)^{4}}\left(\frac{\Lambda}{4 \pi T}\right)^{2 \epsilon} \frac{\lambda(z)}{96 T^{2}}\left(\pi^{2}-6\right),
\end{gathered}
$$




$$
\begin{gathered}
\sum_{\{P\}} \frac{p_{3}^{2}}{p^{4} P^{4}} \mathcal{T}_{p}^{2}=\frac{1}{(2 \pi)^{4}}\left(\frac{\Lambda}{4 \pi T}\right)^{2 \epsilon} \frac{\lambda(z)}{96 T^{2}}\left(-2+\pi^{2}-16 \ln 2\right), \\
\sum_{\{P\}} \frac{p_{3}^{2}}{p^{2} p_{0}^{2} P^{4}} \mathcal{T}_{p}^{2}=\frac{1}{(2 \pi)^{4}}\left(\frac{\Lambda}{4 \pi T}\right)^{2 \epsilon} \frac{\lambda(z)}{288 T^{2}}\left(\pi^{2}-6\right) .
\end{gathered}
$$

[1] Y. Aoki, G. Endrodi, Z. Fodor, S. D. Katz, and K. K. Szabo, Nature (London) 443, 675 (2006).

[2] T. Bhattacharya, M. I. Buchoff, N. H. Christ, H. T. Ding, R. Gupta, C. Jung, F. Karsch, Z. Lin, R. D. Mawhinney, G. McGlynn et al., Phys. Rev. Lett. 113, 082001 (2014).

[3] K. Fukushima, Phys. Lett. B 591, 277 (2004).

[4] C. Ratti, M. A. Thaler, and W. Weise, Phys. Rev. D 73, 014019 (2006).

[5] Z. Fang, Y. L. Wu, and L. Zhang, Phys. Rev. D 99, 034028 (2019).

[6] C. S. Fischer, Phys. Rev. Lett. 103, 052003 (2009).

[7] J. Braun, L. M. Haas, F. Marhauser, and J. M. Pawlowski, Phys. Rev. Lett. 106, 022002 (2011).

[8] F. Karsch and E. Laermann, Phys. Rev. D 50, 6954 (1994).

[9] C. Bernard et al. (MILC Collaboration), Phys. Rev. D 71, 034504 (2005).

[10] M. Cheng, N. H. Christ, S. Datta, J. van der Heide, C. Jung, F. Karsch, O. Kaczmarek, E. Laermann, R. D. Mawhinney, C. Miao et al., Phys. Rev. D 74, 054507 (2006).

[11] L. K. Wu, X. Q. Luo, and H. S. Chen, Phys. Rev. D 76, 034505 (2007).

[12] S. Digal, E. Laermann, and H. Satz, Eur. Phys. J. C 18, 583 (2001).

[13] P. Chakraborty, M. G. Mustafa, and M. H. Thoma, Phys. Rev. D 67, 114004 (2003).

[14] A. V. Smilga and J. J. M. Verbaarschot, Phys. Rev. D 54, 1087 (1996).

[15] P. Zhuang, J. Hufner, and S. P. Klevansky, Nucl. Phys. A576, 525 (1994).

[16] C. Sasaki, B. Friman, and K. Redlich, Phys. Rev. D 75, 074013 (2007).

[17] D. Blaschke, A. Holl, C. D. Roberts, and S. M. Schmidt, Phys. Rev. C 58, 1758 (1998).

[18] D. E. Kharzeev, Prog. Part. Nucl. Phys. 75, 133 (2014).

[19] K. Fukushima, Lect. Notes Phys. 871, 241 (2013).

[20] G. S. Bali, F. Bruckmann, G. Endrodi, Z. Fodor, S. D. Katz, S. Krieg, A. Schafer, and K. K. Szabo, J. High Energy Phys. 02 (2012) 044.

[21] A. Bandyopadhyay, B. Karmakar, N. Haque, and M. G. Mustafa, Phys. Rev. D 100, 034031 (2019).

[22] B. Karmakar, R. Ghosh, A. Bandyopadhyay, N. Haque, and M. G. Mustafa, Phys. Rev. D 99, 094002 (2019).

[23] S. Rath and B. K. Patra, J. High Energy Phys. 12 (2017) 098.

[24] K. Hattori, X. G. Huang, D. H. Rischke, and D. Satow, Phys. Rev. D 96, 094009 (2017).

[25] K. Tuchin, J. Phys. G 39, 025010 (2012).
[26] K. Hattori, S. Li, D. Satow, and H. U. Yee, Phys. Rev. D 95 , 076008 (2017).

[27] M. Kurian, Phys. Rev. D 102, 014041 (2020).

[28] M. Kurian, V. Chandra, and S. K. Das, Phys. Rev. D 101, 094024 (2020).

[29] M. Kurian, S. Mitra, S. Ghosh, and V. Chandra, Eur. Phys. J. C 79, 134 (2019).

[30] M. Kurian and V. Chandra, Phys. Rev. D 97, 116008 (2018).

[31] K. Tuchin, Adv. High Energy Phys. 2013, 1 (2013).

[32] K. Tuchin, Phys. Rev. C 88, 024910 (2013).

[33] A. Bandyopadhyay, C. A. Islam, and M. G. Mustafa, Phys. Rev. D 94, 114034 (2016).

[34] S. Ghosh and V. Chandra, Phys. Rev. D 98, 076006 (2018).

[35] A. Das, N. Haque, M. G. Mustafa, and P. K. Roy, Phys. Rev. D 99, 094022 (2019).

[36] M. Hasan, B. Chatterjee, and B. K. Patra, Eur. Phys. J. C 77, 767 (2017).

[37] B. Singh, L. Thakur, and H. Mishra, Phys. Rev. D 97, 096011 (2018).

[38] R. Ghosh, B. Karmakar, and M. G. Mustafa, Phys. Rev. D 101, 056007 (2020).

[39] I. A. Shovkovy, Lect. Notes Phys. 871, 13 (2013).

[40] G. S. Bali, F. Bruckmann, G. Endrodi, Z. Fodor, S. D. Katz, and A. Schafer, Phys. Rev. D 86, 071502 (2012).

[41] A. Das, D. Kumar, and H. Mishra, Phys. Rev. D 100, 094030 (2019).

[42] A. Bzdak and V. Skokov, Phys. Rev. Lett. 110, 192301 (2013).

[43] L. McLerran and V. Skokov, Nucl. Phys. A929, 184 (2014).

[44] A. Das, A. Bandyopadhyay, P. K. Roy, and M. G. Mustafa, Phys. Rev. D 97, 034024 (2018).

[45] A. Das and N. Haque, Phys. Rev. D 101, 074033 (2020).

[46] T. K. Chyi, C. W. Hwang, W. F. Kao, G. L. Lin, K. W. Ng, and J. J. Tseng, Phys. Rev. D 62, 105014 (2000).

[47] A. Ayala, C. A. Dominguez, S. Hernandez-Ortiz, L. A. Hernandez, M. Loewe, D. Manreza Paret, and R. Zamora, Phys. Rev. D 98, 031501 (2018).

[48] J. Beringer et al. (Particle Data Group), Phys. Rev. D 86, 010001 (2012).

[49] J. I. Kapusta and C. Gale, Finite-Temperature Field Theory: Principles and Applications (Cambridge University Press, Cambridge, England, 2009).

[50] A. Ayala, J. J. Cobos-Martínez, M. Loewe, M. E. Tejeda-Yeomans, and R. Zamora, Phys. Rev. D 91, 016007 (2015).

[51] J. O. Andersen, E. Braaten, E. Petitgirard, and M. Strickland, Phys. Rev. D 66, 085016 (2002). 